\title{
The information de-coupling point
}

\author{
A.J.M. Beulens ${ }^{1)}$, M.H. Jansen 2) , J.C. Wortmann ${ }^{3)}$ \\ 1) Wageningen University, Dreijenplein 2, $6703 \mathrm{HB}$ \\ Wageningen, The Netherland
}

Tel: 31317484460

Fax: 3131748473

e-mail:beulen@rcl.wau.nl

${ }^{2)}$ KPMG, P.O. Box 155, 3454 ZK De Meern, The Netherlands

Tel: 31306581658

Fax: 31306583658

e-mail: jansenvullers.monique@kpmg.nl

${ }^{3)}$ Eindhoven University of Technology, P.O. Box 513, $5600 \mathrm{MB}$ Eindhoven, The Netherlands

Tel: 31402472290

Fax: 31402432612

e-mail:j.c.wortmann@tm.tue.nl

\begin{abstract}
Information systems are usually developed for individual enterprises. Stakeholders in supply networks (i.e. basically all parties involved, but for sure manufacturers, wholesalers, retailers, consumers) are increasingly concerned about properties of products and associated processes or services which are delivered. These properties represent not only measurable physical properties ("does this product contain toxic material?"), but also properties, which are difficult or impossible to measure.

In order to answer these and many other questions, current information systems are usually inadequate. However, an interesting concept is emerging in practice: the information de-coupling point (IDP). This is a point in the supply chain or supply network, where a set of product properties are replaced by a common code that can be referenced downstream in the chain without carrying upstream details from which it was created at the IDP point.

The paper considers possibilities of using information de-coupling points for various purposes. An IDP may create redundant information, which can change over time, and which may not remain to be true over time. Moreover, an IDP has fundamental assumptions on the way in which products can be identified. This
\end{abstract}


point can be illustrated with many examples from quality control, e.g. certification of cars, authentication of documents, etc.

The idea of an IDP is more general than it may seem. For example, the normal idea of a code-number carries a number of implicit assumptions on IDPs. These assumptions are investigated in the paper.

\section{Keywords}

Environmental Information Systems, Product Certification, Product Modeling, Extended Enterprise.

\section{INTRODUCTION}

The notions of an extended enterprise or a supply chain or supply network (chain) raised considerable interest in recent years. For several good reasons, such as globalization of the economy, customization of products, and pressure towards environmentally benign production, customers and suppliers are often included in today's definition of an enterprise. "The object of study should not be restricted to a single plant or production facility, but should include chains or networks of production and physical distribution" (Browne et al. 1995, p.240, Beers et al. 1998).

However, both the notion of the extended enterprise and the notion of a supply network is not very clear. For example, the extended enterprise of a particular company will probably include the company's suppliers, but does it also comprise the suppliers of the suppliers? If so, what are the criteria to create a boundary for the notion of an extended enterprise?

The question of delineation of the extended enterprise is not only of theoretical interest. Many practitioners would argue, that a company's extended enterprise includes suppliers and customers with whom the company has long-lasting relationships. Joint optimization of the business processes and intensive communication becomes possible. But the point of long-lasting relationships between the $1^{\text {st }}$ and $2^{\text {nd }}$ tier supplier is not satisfactory for end-users who want information over the $3^{\text {rd }}, 4^{\text {th }}$ and 5 th tier supplier. Moreover, the same holds for these suppliers who want information on preferences, requirements and experiences of such users.

This paper provides an analysis of these questions from an information modeling point of view. Our basic assertion is that the notion of a supply network or chain is rooted in the fact that:

- Consumers and users of products and services and other stakeholders (e.g. legislators, consumer organizations, etc) require information about the products and its processing history, thus basically identifying a conceptual supply network with necessary roles to be performed.

- In contrast, the notion of an extended enterprise is rooted in the fact that

- Companies realize that in particular contractual and legal obligations may expand over a considerable longer period than the delivery of goods. Partly as 
a consequence they need longer-term relationships with associated partners also regarding information processing and reporting.

More specifically on the second point, the classical concept of a transaction is an agreement between two parties, a supplier and a customer. The supplier delivers specified goods or services, and the customer pays for it (ever since the invention of money). Given this basic notion of a transaction, third parties may be subcontracted to provide support in the process of ordering, delivery or payment. For example, expediters and shippers may serve in transportation of physical goods. Banks may support in the payment process. Agents may help in the ordering process. Insurance companies may transform risks into costs. But all these services do not change the basic nature of a transaction in a classical supply chain.

However, this basic scheme has been changing gradually for several reasons. First of all, legislation has obliged suppliers to give warranty over the quality of their goods. (In ancient Egypt, bakers were punished by the government if they put sand into the bread). Secondly, the need to give a warranty, has led to quite some effort to provide tracking and traceability in many industries. It refers to the ability to document the history of delivered goods and services, and to prove conformance to specifications. However, traceability also implies that a long time after closing the transaction, the customer and supplier still have a relationship.

Thirdly, the importance of steady and guaranteed quality should not be underestimated. It may lead to considerable savings in the transaction costs. If the supplier and customer trust each other, less effort is required by both parties to guarantee and document that the required level of quality is provided. This fact is a basis for establishing longer term relationships between suppliers and customers. It constitutes the first step towards the notion of the extended enterprise.

Fourthly, the supplier may take a next step and offer the customer additional services for mutual interest. For example, the supplier may offer the customer support in dealing with the customer's customer. Currently, many ECR initiatives have this nature.

Finally, the supplier and customer may jointly optimize the offering to the final customer, in which case there is a very strong coupling between partners in the extended enterprise. However, there is no reason why this last model should always be better in any sense of the word than one of the earlier models. Rather, all these forms seem to co-exist currently in a context that is characterized by continuous change.

What can be concluded from the above characterization of extended enterprises? A variety of stakeholders (consumers, retailers, shippers, insurance companies, suppliers, manufacturers, governmental agencies, etc.) have information requirements about products and services. These information requirements may expand across the borders of individual actors in the extended enterprise or the chain or even expand across the borders of the chain as a whole. These information requirements may expand in time, cover the full life cycle of a product instance or product type and they may pertain to different aspects of products and processes. 
A more precise and encompassing analysis of the nature and delineation of extended enterprises requires a more precise analysis of these information requirements. We need concepts, which describe the nature of the stakeholders' information requirements with respect to products and services and in turn help us to design and implement IS for the extended enterprise. One such a concept is the Information Decoupling Point (IDP). It refers to a point in the supply chain, where information about particular orders, goods or processes is aggregated, so that it can be characterized by a certain label or certificate.

In Section 2 of this paper, we will define the information decoupling point, and investigate its nature. Section 3 discusses its usage for various purposes. Section 4 provides examples, mostly taken from current practice. These show, that IDPs can be found in many places. However, their generalized properties have not yet been investigated properly. Section 5 discusses possible contributions of the IDP concept. Section 6 concludes the paper.

NB. We feel that it is beyond the scope of this paper to address consequences for information systems design for partners in the supply chain, nor for the supply chain as a whole. However, we consider this another topic for further research.

\section{THE NATURE OF THE INFORMATION DECOUPLING POINT}

An information decoupling point (IDP) is a point in a supply chain, where a set of product properties is replaced by a common code, such as a certificate. More specifically, an IDP describes in which part of the supply chain properties of products (or services) are described in detail and from where, downstream in the chain, this detailed data is aggregated. Up to the IDP, available data is attached to the product. At the IDP, an IDP-code (unique identification) is attached to the product, and new downstream data may be attached to the product additionally. A more elaborate definition of an IDP is beyond the scope of this paper. We will deal with that in a more extensive paper. Therefore we will now discuss a number of properties of an IDP, in order to explain the concept in more detail.

First of all, in product descriptions, a distinction can be made between product item (often denoted by a code number) and product instance (such as a particular batch or a serial number). The same distinction is known under different names, such as state-independent versus state-dependent data (Bertrand et al, 1991) or typogeny versus ontogeny (Goossenaerts, 1997). An example, a certificate is give to physical materials in a certain state, not to their generic description. However, we will see later in this paper that state-independent data, such as a code number, can serve as a special case of an information decoupling point.

Secondly, an IDP can be conceptualized as a pair of attribute and value, as common in information modeling. This opens the possibility of several related values for the same attribute at a particular point in the supply chain. In case of quality applications, this concept is often used to distinguish different grades of information decoupling point may be related to instances, batches, serialized items, 
and items. For quality. Of course, the notion of an IDP can be generalized to a multi-valued attribute.

Thirdly, an IDP may be used to generate characteristics of the materials to which a value is attached. In one extreme case, this may lead to an IDP that serves as a shorthand for a larger set of attributes and values. For example, an IDP attribute "packaged" with value "tetrapak" may lead to other attributes with values being generated (such as length, width, and height). However, an IDP is usually more than merely a shorthand for other attributes: if a certain liquid is packaged in "tetrapak", we know more than merely length, width, and height. If the IDP stands for the attribute "way of breeding cattle", and it has the value "animal friendly", then it is not self-evident what the generating characteristics are.

Fourthly, it is interesting to note, that a code number for an item is usually considered to be a shorthand for a set of attribute-values without additional meaning: a code number can be used to generate values for a characteristic set of attributes, and a set of values uniquely determines the code number. Usually, there is a responsible actor in the supply chain who is responsible to provide the mappings. Therefore, a code number is a special case of an IDP, as mentioned above.

Fifthly, the notion of a responsible actor is not only relevant in case of code numbers, but even more in case of an IDP which carries warranty or certification. In such cases, a trusted party provides a guarantee that the responsible actor can provide the detailed data on which the IDP value is based. Therefore, data related to particular instance of goods can be traced back upstream.

Finally, it should be noted that different aspect systems my use different IDPs. In principle, such aspect systems as logistics, quality control, human safety and health control, environmentally benign supply, animal friendliness, and many others may all have their own IDPs, and IDPs in different aspect systems do not need to coincide.

\section{USE OF THE IDP FOR DIFFERENT PURPOSES}

First of all, IDPs are useful in determining the delineation of an extended enterprise with respect to information exchange between actors outside and inside the extended enterprise. If the trusted party governs an open market subject to legal rules, then there is no reason to consider the supplier and customer to be part of each other's extended enterprise. However, if there is no such trusted party, or if the responsible actors mutually trust each other because of longer term contracts and mutual relationships, there is reason to consider the supplier and customer as belonging to an extended enterprise.

Apart from the question of a definition of an extended enterprise, IDPs may play an important role in determining information interfaces between actors in the supply chain taking into account various information aspect systems. IDPs may play an important role in arriving at (economically) feasible interfaces between systems. Moreover, explicitly defined IDPs are essential for IS with guaranteed 
integrity of information exchanged and systems that are efficient to operate. It is beyond the scope of this publication to give an in-depth argumentation to support these statements and we suffice to sketch some ideas that support these claimed effects. If we are explicit about defining the syntax and semantics of the information interchange via an IDP at two sides of an IDP-point and that interface is certified then we have reasonable certainty about the integrity of information exchanged. Secondly, by explicitly defining the aggregation to take place at an IDP, we may gain substantial efficiency downstream the IDP since we only have to carry there the IDP-code without loosing the ability to trace back that nonaggregated data when necessary. An ability only invoked when necessary.

The information exchanged may pertain to different types of information flows in supply networks. The notion of an IDP has been introduced as attached to product instances. However, the same notion may be used in other business processes, such as product development, contracting, and ordering.

In product development, market requirements may be stated specifically in terms of properties such as IDPs (see next Section). The same is true in contracting and ordering: the terms used in IDPs refer to more general properties, which may be expected from (potential) customers.

\section{EXAMPLES OF POSSIBLES USES OF IDPS.}

There are a variety of information requirements to be satisfied on the level of interfaces between actors in an extended enterprise. In the sequel we give some examples, but the list is not at all exhaustive. Applications of IDPs may have to do with:

- Product responsibility.

Data have to be stored throughout the supply chain to guarantee that products and associated processes and resources (raw and production resources) meet specifications and legal obligations. Furthermore, in this context we have to do with tracking and tracing requirements to be satisfied. Identification of batches are then of particular importance.

- Product Certification.

Certification is a means to guarantee a particular level of product quality. Such a guarantee can be based on measuring actual values of those characteristics of the end product and or by describing the product history represented by a set of attributes. The latter may pertain to for instance resources used (animal friendliness), process characteristics, etc.

- Reverse Logistics.

We may use IDPs for recycling and for packaging. An IDP may refer to a standard way of packaging and of using packaging materials, which creates more easy recycling of packaging materials. The same is true for recycling in general. For example, an IDP for a bag may state "this product may be recycled in the recycling of paper", etc. If an associated IDP carries a guarantee for integrity of associated properties we have to do again with a certificate, in this case for recycling. 
- Logistics control.

We use a variety of codes to enable us identify and describe a variety of 'forms of appearance' of products that have a number of characteristics in common necessary for efficient logistics operations.

- Material handling.

Basically this type of usage of IDPs has been covered in previous points. However to highlight some specific types of usage of IDPs that are for instance important in agri- and food chains we give some additional examples related to the production/supply of a product. These examples are:

1. The use of tranport means that satisfy certain constraints.

2. The use of GMO free raw materials.

3. The use of allowed financial resources.

- Animal friendliness

The consumer in modern society is sensitive to guarantee animal friendliness in the supply chain of food. This is currently typically solved by introducing marketing labels. Consumer organizations and/or legal requirements enforce companies to be able to prove whatever is stated in these labels. The concept of an IDP is the only efficient and effective way of realization of these requirements.

- Human health care

For human health care, the situation is analogous to the situation of animal friendliness. Here, too, the consumer wants guarantees regarding raw materials used and processing steps before usage or consumption. The notion of an IDP is crucial in guaranteeing the required properties.

- Information to the customer.

Also in case of providing information to customers regarding products and their properties IDPs may come in handy. IDPs may be used to convey generic information pertaining for example to:

1. The way of preparation.

2. Specific recipe information with raw materials used.

3. Composition information. E.g. Total \% sugar in Jam from apples and beet sugar together.

4. Attributes related to allergies of people.

NB. All previous examples may also be seen in the context of certification.

\section{DISCUSSION}

The previous descriptions of the concept of IDPs and examples of possible usage indicate that:

- The concept is already used frequently for a number of applications encompassing product identification on different levels, for certificates or labels and to a certain extend for recycling and customer information.

- The concept may contribute to our opinion to clearly define explicitly information interfaces between supply chain actors and between a supply chain and its environment. Interfaces with defined and shared syntaxis and 
semantics necessary to understand each other unambiguously. As a result guarantees with respect to the integrity of information exchanged and with respect to the abilities of responsible actors related to tracking and tracing can be given.

- Using IDPs may substantially reduce the volumes of information to be processed downstream in the supply chain when tracking and tracing is at stake. This is due to the aggregation that takes place. The combination of increasing information requirements on the level of the supply chain (or the extended enterprise) and the appropriate use of IDPs may contribute to satisfying these requirements with guaranteed integrity while satisfying economic and operational feasibility of necessary IS. These claims are currently only supported by limited experiences obtained in designing environmental IS and by the usage of shared EAN codes in retail chains.

\section{CONCLUDING REMARKS}

In this paper we have described the concept of IDPs that has been developed in the context of environmental IS. Based on limited design experience we have found it to be a valuable concept for delineating extended enterprises from their environment and for designing IS for extended enterprises and supply chains. Since we report about 'research in progress', many issues have not yet been adequately dealt with and many questions have yet to be investigated. One of these questions has to do with integrating different aspect IDPs at one IDP interface point into one encompassing one. Another question is related to an in-depth formal treatment of the concept. Some of these issues will be discussed in a more extensive paper.

\section{REFERENCES}

Beers, G., Beulens, A.J.M. and. Van Dalen, J.Chr: (1998)

Chain Science as an emerging discipline, in Proceedings of the third international conference on chain management in the agribusiness and the food industry, (eds.: Ziggers, G.W. Trienekens, J.H. Zuurbier P.J.P.) Wageningen Agricultural University, ISBN: 90-6754-528-7.

Bertrand, J.W.M, Wortmann, J.C and Wijngaard, J.(1991)

Production Control - a structural and design-oriented approach.

Elsevier, Amsterdam.

Browne, J., Sackett, P. and Wortmann, J.C. (1995)

Future manufacturing systems -- Towards the extended enterprise, in Computers in Industry, 25, 235- 254.

Goossenaerts, J.(1997)

A Framework for Connecting Work and Information Infrastructure, in

Information Infrastructures in Manufacturing, (eds. J. Goossenaerts, F.Kimura and J.C. Wortmann): Chapman \& Hall. 


\section{BIOGRAPHY}

Prof.dr.ir. J.C. Wortmann joined Eindhoven University of Technology as a full professor in industrial information systems in 1986. He has been active in the development of logistics control systems since his graduation in 1974. He was one of the first professionals in this field. He developed logistics control systems for Philips Components and he wrote a doctoral thesis on shop floor control systems in 1981.

He has been the overall project manager of the ESPRIT Basic research action 3143 "Factory of the Future". He has acted as a reviewer for several programs and projects of the CEC. He is currently editor in chief of the international journal "Computers in Industry". $\mathrm{He}$ is actively involved in many research projects together with Baan. 